\title{
Ansatzpunkte für eine Theologie des Neuen Testaments bei Oscar Cullmann und Leonhard Goppelt
}

\author{
PJ Grăbe \\ Universiteit van Suid-Afrika
}

\begin{abstract}
Points of departure for a theology of the New Testament: Oscar Cullmann and Leonhard Goppelt
\end{abstract}

Both Cullmann and Goppelt offer alternative positions to an existential approach to New Testament theology. After a consideration of Oscar Cullmann's position in the history of New Testament theology, special attention is given to his concept of salvation history, as well as a critical evaluation of this concept. Goppelt associates himself with the hermeneutical point of departure of Cullmann and Von Rad. Salvation history is, however, filled with new content through reflection on the earthly Jesus. Goppelt's starting point for a theology of the New Testament is not found in a general easter 'kerygma', but in the unfolding of Jesus' words and deeds.

\section{OSCAR CULLMANN}

1.1 Die Position von Oscar Cullmann in der Geschichte der neutestamentlichen Theologie

'Die Zeit um die Jahrhundertwende ist durch die religionsgeschichtliche Blickweise oder aber - im konservativen protestantischen Lager - durch positiv-biblische Darstellungen gekennzeichnet' (Schnackenburg 1963: 25). Der Vorstoß über die positiv-biblische und die religionsgeschichtliche Darstellungsweise hinaus erfolgte

- Gegründet auf ein Referal gehalten bei dem 'Seminar für Exegese des Neuen Testaments' (Oberseminar), Westfalische Wilhelms-Universität Münster. 
(só Schnackenburg 1963: 29) vor allem in einer zweifachen Richtung.

Die einen erblicken die theologische Leitlinie, die den bisherigen Werken fehlte, im biblischen Gedanken der Heilsgeschichte, die anderen schauen die neutestamentliche Theologie von der heutigen theologischen Problematik her an und wollen sie dem modernen Menschen zugänglich machen, namentlich von der Existenztheologie her (vgl. Hasel 1978: 82-102; 111-132).

\subsubsection{Die heilsgeschichtliche Betrachtungsweise}

Schnackenburg (1963: 29) weist mit Recht darauf hin, daß die heilsgeschichtliche Betrachtungsweise nicht neu ist. Im vergangenen Jahrhundert pflegte sie der Erlanger Theologe J Chr $\mathrm{K}$ von Hofmann, dessen 'Biblische Theologie des Neuen Testaments' posthum veröffentlicht wurde. Ansätze dazu sind auch in dem Doppelwerk A Schlatters über die 'Geschichte des Christus' und die 'Theologie der Apostel' zu erkennen. Eine konsequente heilsgeschichtliche Grundkonzeption findet man in dem Lehr- und Arbeitsbuch von E Stauffer. Hauptvertreter der heilsgeschichtlichen Theologie aber ist O Cullmann. Er hat zwar kein Lehrbuch der neutestamentlichen Theologie, aber eine Fülle einschlägiger Arbeiten geschrieben. Für diese Artikel ist sein Buch, 'Heil als Geschichte: Heilsgeschichtliche Existenz im Neuen Testament', von besonderer Bedeutung.

Die theologische Arbeit Von Rads ist auch für Cullmann sehr wichtig, besonders das Verhältnis zwischen Von Rads traditionsgeschichtlicher und Cullmanns heilsgeschichtlicher Methode (Hasel 1978: 116). Manfred Oeming (1985: 25) hat diesen traditionsgeschichtlichen Gesichtspunkt in der Theologie Von Rads gut zusammengefaßt: 'Es hatte sich beim exegetischen Durchgang durch das Alte Testament gezeig1, daß Israel in jeweils neuer Situation die alten Traditionen nicht einfach fallengelassen und durch neue ersetzt hat. Vielmehr hielt Israel durch seine Geschichte hindurch an alten Traditionen fest, aktualisierte sie aber im Lichte neuer Heilssetzungen Jahwes je und je neu und anders.'

\subsubsection{Cullmann als Theologe mit einem ōkumenischen Interesse}

Es sei darauf hingewiesen, daß Cullmann ein ökumenisches Interesse hat. In dem Vorwort zu seinem Buch, 'Heil als Geschichte', lenkt er (1967: vii) die Aufmerksamkeit darauf hin, da $B$ Papst Paul VI vor den nichtkatholischen Beobachtern am Zweiten Vatikanischen Konzil erklărte, daß 'eine konkrete und historische, auf die Heilsgeschichte konzentrierte Theologie' gemeinsame Grundlage des oekumenischen Dialogs sei.

Die Position Cullmanns ist sehr nuanciert. Es ist deshalb wichtig, da $\beta$ eine kurze Erklärung des Begriffs, 'Heilsgeschichte' in seiner Theologie gegeben wird. 


\subsection{Der Begriff 'Heilsgeschichte' in der Theologie Oscar Cullmanns}

\subsubsection{Ereignis und Deutung}

Um den Begriff 'Heilsgeschichte' bei Cullmann zu verstehen, ist es sehr wichtig, daß das Verhältnis zwischen 'Ereignis' und 'Deutung' erklärt werden muß.

In diesem Zusammenhang stellt Cullmann (1967: 70) die Frage: 'Wie entsteht die heilsgeschichtliche Sicht im Alten Testament?' Cullmann stimmt Von Rad darin zu, daß die geschichtliche Überlieferung lsraels, die Geschichtstheologien des Jahwisten, Elohisten, Deuteronomisten und der Propheten sich weiterentwickelt haben, und zwar in der Regel im Zusammenhang mit jeweils neuen Ereignissen, die sich in der Gegenwart dieser Zeugen zugetragen haben. Von den früheren Ereignissen fällt Licht auf die neuen, aber umgekehrt fällt auch ein ganz neues Licht von diesen auf jene. Heilsgeschichte entsteht also nicht durch bloße Addition von Geschehnissen, die im Glauben als Heilsereignisse erkannt sind, sondern jedesmal werden zugleich an der Interpretation der vergangenen Heilsereignisse Korrekturen im Licht der neuen vorgenommen (1967: 71).

Wie im Alten Testament handelt es sich auch im Neuen um ständige Weiterentwicklung des Kerygmas im Zusammenhang mit neuen Ereignissen, um die fortschreitende Verbindung von Gegenwartsreignissen, die von den Zeugen erlebt werden, mit einem schon vorhandenen Kerygma und um dessen damit gegebene Neuinterpretation (Cullmann 1967: 81).

Trotzdem liegen bedeutsame Unterschiede zwischen dem Alten und dem Neuen Testament vor. Das Gegenwartsereignis, das im Neuen Testament Ausgangspunkt der Neuinterpretation ist, wird als das entscheidende Ereignis und als Norm der ganzen Heilsgeschichte angesehen.

Das Reden Gottes im Sohne 'am Ende der Tage' (Heb 1: 2) ist anderer Art als sein mannigfaches Reden 'in den Propheten'. Aber es gehört eben doch in seiner Vertikalität gerade in das horizontale Heilsgeschehen hinein (1967: 82).

Cullmanns Heilsgeschichtlichen Ansatz kann man deutlich bemerken, wenn er (1967: 86) schreibt:

Jetzt wurde ihnen [d h : den Jüngern] nachträglich klar, daß schon damals alles, was sie gesehen und gehört hatten, Leben und Verkündigung Jesu, Offenbarung und zwar die entscheidende Heilsoffenbarung Gottes gewesen war. Es wurde ihnen klar, daß das 
von Jesus gelebte Leben und die von ihm verkündete Lehre zusammen das zentrale Kerygma darstellen, in dem alle Heilsgeschichte gipfelt. Sie hatten zugleich jenes vorgegebene Kerygma Jesu zu überlieferm und neu zu interpretieren.

Er lenkt die Aufmerksamkeit darauf (1967: 86), daß mit dem historischen Jesus nicht nur die Ereignisse gegeben waren, sondern in Jesu Verkündigung bereits ihre heilsgeschichtliche Deutung. Der johanneische Begriff der 'geistgewirkten Erinnerung' ist wichtig für den Zusammenhang von Überliefertem und Neuinterpretiertem. Jesus offenbart sich selbst als den Höhepunkt allen Geschehens: Weil durch ihn ganz bestimmte Dinge geschehen sind, ist das Reich Gottes in seiner Person gekommen (Mt 12: 28-30).

Auf Grund der allgemeinen Charakteristik der fortschreitende Entwicklung des heilsgeschichtlichen Kerygmas im Zusammenhang mit neuen Ereignissen weist Cullmann (1967: 88) darauf hin, daß es eine Entwicklung auch von der Deutung Jesu zur Deutung der Jünger auf Grund der Auferstehungsereignisse gibt. Es gibt aber auch eine Kontinuität zwischen dem Kerygma des 'historischen Jesus' und dem der Jünger.

\subsubsection{Glaube und Heilsgeschichte}

Für ein gutes Verstehen der Heilsgeschichte bei Cullmann muß das Verhältnis zwischen Glaube und Heilsgeschichte geklärt werden. Auf die Frage, ob die heilsgeschichtliche Ereignisfolge für die biblischen Zeugen Gegenstand eines Glaubensaktes ist, antwortet Cullmann (1967: 98), daß es zur Heilsgeschichte gehört, daß der Offenbarungesprozeß in sie einbezogen wird. Aus diesem Grunde ist der Glaube der ersten Zeugen zugleich Glaubensentscheidung für ihre eigene Einreihung in ein zusammenhängendes Geschehen.

Es gehör geradezu zum Wesen des Glaubens im Neuen Testament, auch sofern er von den Lesern verlangt wird, daB er sich auf Ereignisse bezieht, deren Urheber gerade nicht sie sind, auch nicht mit ihrem Glauben, sondern die extra nos geschehen sind: pro nobis, aber extra nos (Cullmann 1967: 101).

Cullmann (1967: 102) weist darauf hin, daß, obwohl die Hörer und Leser der biblischen Zeugen nicht in gleicher Weise mit ihrer eigenen Einreihung am Heilsgeschehen selbst beteiligt sind wie die Propheten und Apostel, doch auch von ihnen diese Glaubensentscheidung verlangt wird. 
Er (1967: 294) macht darauf aufmerksam, daß, wenn wir von unserem Nachvollzug des Glaubens der biblischen Zeugen an dieser Heilsgeschichte sprechen, wir gleichzeitig berücksichtigen müssen, was uns mit jenen Zeugen verbindet und was uns von ihnen trennt.

Meines Erachtens hat Cullmann (1967: 294) recht, wenn er behauptet, daß die falsche Meinung abzulehnen sei, als sei das Trennende in dieser Hinsicht durch den Fortschritt der modernen Wissenschaft bedingt, als könnten wir heute deshalb nicht an die Heilsgeschichte glauben, weil unser Weltbild ein anderes geworden sei.

Das Skandalon des Kreuzes und der Auferstehung als Zentrum allen Geschehens war damals - wie heute - eine "Torheit" ... Wir müssen den Mut haben, das, was Paulus als Skandalon bezeichnet, als das uns wie den Menschen des 1. Jahrhunderts verkündete Glaubensobjekt in seiner Fremdheit stehenzulassen ... (1967: 295).

Wenn Cullmann weiter ausführt, daß es nicht geschehen dürfe, daß unter Berufung auf meinen Glaubensvollzug und meine Glaubensentscheidung doch wieder ich selber - wenn auch in der 'hermeneutischen' Ich-Du-Beziehung - Ausgangspunkt des Heilsgeschehens werde, ist es klar, daß er auf die Theologie Bultmanns anspielt. Cullmann gibt zu, daß mein Glaubensakt und meine Entscheidung unerläßlich sind. Er betont aber, daß, wenn diese als Begegnung unabhängig von einem vorhergehenden ontischen Geschehen verselbständig werden, ich doch wieder im Vordergrund des Heilsgeschehens stehe.

Die Kritik, die Cullmann an der Auffassung Bultmanns übt, trifft meines Erachtens in dieser Hinsicht genau zu. In der Theologie Bultmanns findet man die Identifikation des Glaubens mit dem wahren Verstehen seiner selbst. Diese Identifikation leuchtet nur dann ein, wenn man mit Bultmann das Wesen des Menschen, sein 'eigentliches Dasein', als 'ein Seinkönnen' bestimmt, und gegen den in der aristotelischen Tradition behaupteten ontologischen Primat der Wirklichkeit vor der Möglichkeit, also den ontologischen Primat der Möglichkeit vor der Wirklichkeit vertritt, wobei man sich den Auffassungen des Cusanus, Schellings und Kierkegaards mit Heidegger und auch Bloch anschließt. Bultmann sieht also den Menschen als Wesen der Möglichkeit, als existierende Transzendenz überschwingend in Möglichkeiten, als ein 'Wesen der Ferne' (Jüngel 1985: 54-55).

Die Konzentration des Sich-selbst-Verstehens auf das Verstehen des Augenblicks als eines Augenblicks der Entscheidung erweckt theologisch deshalb Bedenken, weil sie Gefahr läuft, den indikativischen Charakter der christlichen Verkündigung zugunsten des appellativen Moments zu vernachlässigen. Die neu- 
testamentlichen Texte lassen erkennen, da $\beta$ die Entscheidung, bevor sie gefordert wird, durch die indikativische Erzählung der Geschichte Jesu Christi (und der sie implizierenden Geschichten) allererst ermöglicht, und zwar so ermöglicht wird, daß sich die rechte Entscheidung daraufhin in der Regel von selbst versteht (Jüngel 1985: 71-72).

Zum Glauben komme es, só Cullmann (1967: 298), wenn ich von der Heilsgeschichte so überwältigt werde, da $B$ ich nicht anders könne, als mich in sie einbeschlossen zu sehen und mich deshalb bewuBt in sie einzureihen. Christus ist Geschichte geworden, damit alle Geschichte zur Heilsgeschichte werde (1967: 301).

Nachdem er darauf hingewiesen hat, daß der Terminus 'Heilsgeschichte' in der gegenwärtigen Theologie sehr umstritten und mit mancherlei Mißverständnissen belastet ist, schreibt Kasper (1970: 94), daß der Ausdruck 'Heilsgeschichte' keine illegitime Objektivierung des Heils darstelle, denn das Heil werde dadurch ja noch nicht feststellbar für jeden, der offene Augen habe. Die Redeweise von 'Heilstatsachen' solle man unterlassen. Das Heil ist keine Sache, die einfach festgestellt werden kann. Wenn Kasper in diesem Kontext auf die Bedeutung des Wortes Nachdruck legt, bringt er eine Perspektive nach vorne, die meines Erachtens nicht genügend von Cullmann betont wird. Kasper schreibt zu Recht, daß Offenbarung nicht nur Geschichte ist, sie darf aber ebensowenig in ein bloßes Wortgeschehen und ein Sprachereignis aufgelöst werden.

Sosehr das Heil sich innerhalb der Geschichte ereignet, muß es als Heilsereignis doch immer erst im Wort bezeugt werden und kann nur im Glauben an das verkündende und bezeugende Wort angenommen werden (Kasper 1970: 94-95).

\subsubsection{Kontinuität und Veränderung}

Zwei sehr wichtige Themen in der Theologie Cullmanns sind Kontinuität und Veränderung; man könnte auch sagen: Veränderung in der Kontinuität. Immer wieder verändert sich die Perspektive. Die Perspektive weitet sich schrittweise mit jedem neuen Ereignis aus. Das Fortschreiten der Heilsgeschichte und der heilsgeschichtlichen Offenbarung bedeutet Veränderung und Korrektur, doch bleibt dabei die Treue Gottes gewährleistet (1967: 105).

\subsubsection{Neutestamentliche Heilsgeschichte und Geschichte}

Cullmann (1967: 132) weist darauf hin, daß es so scheinen könne, als ob abgesehen von der Ur- und Endgeschichte die biblische Geschichte nun doch Geschichte schlechthin wäre. Es ist deshalb wichtig, daß etwas mehr gesagt wird über das 
Thema 'Neutestamentliche Heilsgeschichte und Geschichte'.

Eine profane Geschichtsschau kommt anders zustande als die biblische. Was Geschichte und Heilsgeschichte ihrer Entstehung nach unterscheidet, ist die Rolle, die in der Heilsgeschichte die Offenbarung sowohl im Erleben der Ereignisse und Tatsachen als auch im gläubigen Aufnehmen der Berichte und ihrer Deutung ('Kerygma') spielt (1967: 133).

Heilsgeschichte ist nicht eine Geschichte neben der Geschichte, sondern sie wickelt sich in der Geschichte ab und gehört in diesem Sinne zu ihr. Es gehört aber zum Wesen der biblischen Heilsgeschichte, daß sie vom historischen Standpunkt aus merkwürdige Lücken aufweist und sich ganz und gar sprunghaft abwickelt. Einzelne Ereignisse erscheinen hier aus dem Gesamtgeschehen - historisch gesprochen willkürlich ausgesondert und ausgezeichnet, und doch besteht zwischen ihnen ein Zusammenhang (Cullmann 1967: 135). Aussonderung und Zusammenhang der Ereignisse, in denen Gott Heil geschehen läßt, beruhen auf dem völlig irrationalen und nicht zu begründenden theologischen Gedanken der göttlichen Erwählung.

Es sei darauf hingewiesen, daß wir es in der Heilsgeschichte mit einem Ineinander von Konstanten, nämlich dem göttlichen Plan, und von Kontingenz, und zwar der Entwicklung der von diesem Plan aus nicht vorauszusehenden göttlichen Einzelereignisse, vor allem auch des Widerstands gegen Gottes Plan zu tun haben (1967: 139).

Cullmann (1967: 141) ist der Meinung, daß eine progressive göttliche Redukrion das Prinzip aller biblischen Heilsgeschichte ist: Schöpfung - Menschheit - Israel Rest - der Eine - und weiter der Weg wiederum zur Vielheit zurück: Apostel Urgemeinde - Kirche aus Juden und Heiden - Welt. Diese göttliche Reduktion hat aber von Anfang an die ganze Menschheit im Auge. Je enger der Rahmen der Heilsgeschichte wird, um so weiter strahlt die heilsgeschicht-liche Wirkung aus.

\subsubsection{Gegenwart und Zukunft}

Eine des wichtigsten Aspekte muB aber noch geklärt werden: das Verhältnis zwischen Gegenwart und Zukunft. In dieser Zusammenhang schreibt Cullmann (1967: 147): 'Die heilsgeschichtliche Spannung zwischen "schon" und "noch nicht" ist der Schlüssel zum Verständnis der neutestamentlichen Heilsgeschichte'.

Das Neue im Neuen Testament ist nicht die Eschatologie, sondern die Spannung zwischen dem entscheidenden 'schon erfült' und dem 'noch nicht vollendet'. Cullmann (1967: 153) ist der Meinung, daß die ganze Theologie des Neuen Testaments und auch schon die Verkündigung Jesu durch diese Spannung bestimmt ist. Das, was man den Enthusiasmus der Urgemeinde nennt, ist nicht durch die Naherwartung als solcher, sondern durch das sie begründende 'Schon' 
hervorgerufen. Die Naherwartung ist nur ein Symptom.

In diesem Zusammenhang zitiert Cullmann (1967: 153) unter anderem Lukas 10: 18: 'Ich sah Satan wie einen Blitz vom Himmel fallen'. Bei allen Unterschieden finden alle Bücher des Neuen Testaments sich in diesem Nebeneinander von 'schon' und 'noch nicht' zusammen.

Er betont (1967: 153), daß an diesem Punkt kein Bruch zwischen Jesus und der Urkirche vorliegt, trotz aller nicht zu übersehenden Unterschiede, und dies trennt zugleich Jesus und die Urkirche vom Judentum.

Die Entscheidungsschlacht ist geschlagen, aber der Krieg geht noch eine unbestimmte Zeit weiter. Die konkrete Auswirkung dieser Zwischenzeit äußert sich nach dem Neuen Testament darin (só Cullmann 1967: 163), daß es die Zeit des 'schon' alles erneuernden endzeitlichen Heiligen Geistes ist (arrabón, aparché) die Zeit der Kirche, die Zeit der Predigt des Evangeliums an die Welt, bevor das Ende kommt.

\subsection{Kritische Bemerkungen}

Der Begriff 'Heilsgeschichte' wurde in den letzten zwei Jahrzehnten stark kritisiert. (Vgl. Clemons 1972: 89: 'Salvation history as a hermeneutical method has dominated biblical studies and theology for more than three decades. Today that dominance is being severely challenged from several quarters'). In seinem Artikel 'Bibel und Heilsgeschichte: Die Fragwürdigkeit einer Idee'(1971: 1-47) vertritt Günther Klein zwei Thesen: 1) Die Resistenz der Bibel gegen die Idee der Heilsgeschichte ist historisch verifizierbar; 2) Die Resistenz der zentralen urchristlichen Konstruktion des Glaubens gegen die Idee der Heilsgeschichte ist theologisch nicht überholbar.

Schon Rudolf Bultmann (1967: 356-368) aüßerte scharfe Kritik gegen die heilsgeschichtliche Methode. Er hielt Cullmann vor, daß er die verschiedenen neutestamentlichen Schriften sozusagen auf die gleiche Fläche auftrage, eine Handlungsweise, die zu einer unerlaubten Harmonisiering führe. Obwohl die Idee der Heilsgeschichte eine Rolle spiele bei Paulus, im Hebräerbrief, bei Matthäus und in gewisser Weise auch bei Lukas und in der Apostelgeschichte (gewiß aber nicht bei Johannes), sei es eine weit übertriebene Behauptung, da $B$ das ganze Neue Testament eine einheitliche Auffassung der Heilsgeschichte voraussetze (vgl.1967: 364).

Weil das religionsgeschichtliche Problem von Cullmann ignoriert werde, só Bultmann, werde der Einfluß der jüdischen Apokalyptik auf das neutestamentliche Verständnis der Heilsgeschichte unterschätzt. Die christliche Geschichtsphilosophie, die Cullmann entwirft, 'ist nichts anderes als die jüdisch-apokalyptische 
Spekulation, modifiziert nur dadurch, daß sich die "Mitte" nach rückwärts verschoben hat' (1967: 365).

Bultmann ist der Meinung, da 3 nach urchristlichen Denken Christus vielmehr das Ende der Geschichte und Heilsgeschichte ist (nicht die Mitte der Geschichte, wie Cullmann behauptet). 'Hinfort kann es keine Geschichte mehr geben, auch keine Heilsgeschichte ...' (1967: 336).

Fuchs (1960: 91) gibt zu, daß Cullmann vielleicht schärfer als sogar Schweitzer sehe, daß Jesu Kreuz ein heilsgeschichtliches Faktum der Vergangenheit ist. Aber Cullmann sehe nicht, só Fuchs, daß Jesu Kreuz selber das Eschaton sei, '... der Satz, Christus ist des Gesetzes Ende, hat den eschatologischen Grund, daß Christus am Kreuz das Ende der Geschichte war ...' (1960: 91).

In seiner Monographie, 'Abschied von der Heilsgeschichte', wirft Franz Hesse (1971) der Theologie der Heilsgeschichte vor, daß sie das von Gott gesetzte Heil nicht als den Punkt sehe, der er faktisch sei, allein gegeben und gesetzt in dem Christus crucifixus. Wo nur ein Punkt ist, sieht es wie eine Linie aus, die sich aus vielen anscheinend gleich wichtigen Punkten zusammensetzt, und so entsteht eine ganze Heilsgeschichte. Weil aber das Heil, das Glaube und Theologie meinen, só Hesse (1971: 66-67), nirgendwo in der Geschichte 'enthalten' sei, weil die Geschichte darum auch nicht auf dieses Heil hinführen könne, sollte man endlich Abschied nehmen von dem Theologumenon der Heilsgeschichte.

Es gibt aber auch Forscher, die sich gegen die obengenannte abwertende Haltung gegenüber der Heilsgeschichte im Neuen Testament wenden, indem sie die durchgehende und wesentliche Rolle der Heilsgeschichte in der Entwicklung des neutestamentlichen Denkens betonen (Kümmel 1978: 164 Anm 43). Ernst Käsemann weist darauf hin, $\mathrm{da} ß$ man das Wort 'Heilsgeschichte' nicht aus der mit ihm verbundenen Problematik herausnehmen solle und möglichst genau defmieren müße. Jedoch schreibt er (1972: 112): 'Ich habe nichts gegen das Wort "heilsgeschichtlich" ... und meine sogar, da $B$ man die Bibel in allgemeinen und Paulus in besonderem ohne die heilsgeschichtliche Perspektive nicht begreifen kann.' Käsemann ist der Ansicht, daß der heilsgeshichtliche Horizont der paulinischen Theologie sich nicht ernsthaft bestreiten lasse. Jedoch müßten wir danach fragen, wie Heilsgeschichte vom Apostel Paulus verstanden wird und welche Funktion sie im Ganzen seiner Theologie hat (1972: 118-119).

GE Ladd (1971: 62) verteidigt die heilsgeschichtliche Betrachtung der Bibel als eine sachgemäße Methode der Darstellung biblischer Theologie: '... revelation through the deed-word complex of Heilsgeschichte may be taken as the main theme of the Bible'. 
Die Frage besteht, wie weit Cullmann sich am ganzen Neuen Testament orientiert, oder nur an einem besonderen Aspekt, nämlich an Lukas (Conzelmann 1964; Vielhauer 1965: 23-26) oder an einem lukanisch interpretierten Paulus.

Goppelt (1985: 387) ist der Meinung, daß die typologischen Entwürfe in Römer 4 und Römer 5: 12-21 nicht Ausschnitte aus einem universalen Heilsplan sind. Paulus präge die Begriffe é $\pi \alpha \gamma \gamma \in \lambda i \alpha$ und túmoc, aber er entwickele keinen Begriff für 'Heilsplan' oder 'Heilsprozeß'. Das Wort oikovouía, eigentlich 'Hausverwaltung', nehme erst in Epheser 1: 10, 3: 2 und 9 ansatzweise die Bedeutung 'Heilsplan' an, in der es in der Theologie des 2. Jahrhunderts gebraucht werde. Erst bei Irenäus werde er Leitbegriff für den heilsgeschichtlichen Proze $B$, in dem Christus, ähnlich wie bei Cullmann, als 'die Mitte der Zeit' stehe. Der apokalyptische Begriff

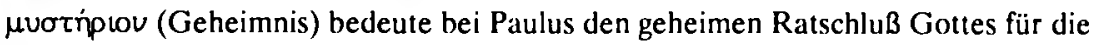
Endzeit, nicht aber einen Geschichtsplan. Paulus kenne 'Heilsgeschichte' nicht als geschichtlichen Prozeß. Er kennt Heilsgeschichte als Erwählungs-, Verheißungsund Berufungsgeschichte.

Auch Otto Merk (1972: 253) meint, daß Cullmann in der 'Heilsgeschichte' nur Teilaspekte der neutestamentlichen Theologie behandele.

Obwohl die Gefahr besteht, bei der Verwendung des Begriffs 'Heilsgeschichte' die verschiedenen neutestamentlichen Schriften auf die gleiche Fläche aufzutragen (was Bultmann Cullmann vorhielt), meine ich jedoch nicht, daß man (wie Hesse plädierte) von der Heilsgeschichte Abschied nehmen solle. Obwohl die Kritik an der Theologie Cullmanns unterstreicht, daß die Aufgabe einer Theologie des Neuen Testaments nicht mit einer Ausarbeitung der Heilsgeschichte erschöpft ist, bin ich jedoch der Meinung, daß mit Schnackenburg gesagt werden muß: '...es wird sich nicht leugnen lassen, daß die Heilsgeschichte eine Grundkategorie biblischen Denkens ist, die das spannungsreiche Verhältnis von "Verheißung und Erfüllung" vom Sündenfall bis zur Endvollendung durchträgt’ (auch Kümmel 1978: 176).

\section{LEONHARD GOPPELT}

Jürgen Roloff, der die Theologie von Goppelt herausgegeben hat, schreibt in seinem Vorwort (1985: 5): 'Mehr als ein Jahrzehnt lang war für Leonhard Goppelt die Arbeit an seiner Neutestamentlichen Theologie die alles beherrschende Mitte ... bis zu seinem frühen Tode am 21. Dezember 1973'.

Den ersten Band seiner Theologie hinterließ Leonhard Goppelt als weitgehend fertiges Manuskript. Obwohl der zweite Band nur in seinen wesentlichen Teilen von Goppelt abgeschlossen werden konnte, hat Jürgen Roloff versucht, Goppelt selbst so klar wie möglich zu Wort kommen zu lassen. 


\subsection{Einführung und Aufgabestellung}

Zur Einführung seiner Theologie schreibt Goppelt (1985: 17), daß das Neue Testament die einzigen zuverlässigen Überlieferungen über das Auftreten Jesu wie über das grundlegende Werden der Kirche und ihrer Verkündigung enthält.

Die Schriften des Neuen Testaments aber sind durchweg Worte für bestimmte geschichtliche Situationen. Aus den einzelnen Schriften oder Schriftengruppen sachlich geordnete zusammenhängende Biider des Wirkens Jesu oder der Verkündigung und Lehre in der ersten Kirche zu gewinnen, ist das Ziel einer "Theologie des Neuen Testaments" (Goppelt 1985: 17).

Er ist der Meinung (1985: 18), daß wir das Verständnis des Neuen Testaments ebensowenig statischen Denkvoraussetzungen der Moderne unterwerfen und umgekehrt den Menschen und die Gesellschaft von heute lediglich mit dem 'Buchstaben' neutestamentlichen Aussagen konfrontieren dürfen. Beide, das Neue Testament und die Menschen von Heute, müssen miteinander in einen kritischen Dialog gebracht werden.

Am Anfang seines Buches gibt Goppelt eine umfangreiche Diskussion der Geschichte und des Problemfelds der Disziplin. Er gibt Informationen über die Entstehung der Disziplin, die Entwicklung der 'rein historischen' Fragestellung und ihre Ergebnisse, die theologische Erweiterung der 'rein historischen' Fragestellung, die historisch-positive Richtung und die 'heilsgeschichtliche' Richtung historischer Schriftforschung. Besonders wichtig für Goppelt ist die Arbeit Oscar Cullmanns und Gerhard von Rads. Er schreibt (1985: 50): 'Wir suchen ihn [unseren hermeneutischen Ansatz] in Weiterführung der zuletzt charakterisierten dritten Möglichkeit der Fragestellung', (das heißt bei Oscar Cullmann und Gerhard von Rad). Goppelt stellt sich die Aufgabe, das Prinzip der historisch-kritischen Schriftforschung, Kritik, Analogie und Korrelation mit dem Selbstverständnis des Neuen Testaments in einen kritischen Dialog zu bringen. Er formuliert (1985: 50): 'Als Ergebnis des intendierten kritischen Dialogs suchen wir ein historisch-kritisch reflektiertes und zugleich sachlich verstehbares Bild der Ntl. Theologie ... zu gewinnen ....'

2.2 Die Theologie Gerhard von Rads: Anschließung und Weiterführung durch I conhard Goppelt

Weil Goppelt die Linie Von Rads aufgreift und weiterführt, ist es nötig etwas über die Theologie Von Rads zu sagen: Er versucht, das Denkmodell 'Verheißung Erfüllung' im Horizont der historisch-kritischen Methode zu erneuern. Die 
Theologie Von Rads hat eine Offenheit.

... das Alte Testament läßt sich nicht anders lesen als das Buch einer ständig wachsenden Erwartung ... Aber merkwürdigerweise war keine Erfüllung in der Geschichte imstande, diese Erwartung zu befriedigen und zur Ruhe zu bringen ... So ist die Geschichte des Jahweglaubens charakterisiert durch immer neue Zäsuren, durch immer neue Einbrüche von göttlichen Setzungen, durch Neubeginne, die in traditionsgeschichtlicher Hinsicht neue Perioden einleiten (Von Rad 1968: 339-340).

Von Rad (1968: 341) weist darauf hin, daß die historisch-kritische Exegese keine sichere Antwort geben kann auf die Frage, ob das Neue Testament als Erfüllung der Erwartung des Alten Testaments gesehen werden muß oder kann. Jedoch ist er der Meinung, daß der christliche Ausleger sich nicht auf das beschränken dürfe, was mit den Mitteln der 'neutralen' Exegese an Wahrheit ermittelt werden kann. Vielmehr muß er seinen christlichen Glauben in die Auslegung miteinbeziehen, um zur Erkenntnis des vollen Wahrheitsgehalts des Alten Testaments zu gelangen.

Manfred Oeming (1985: 20-33) hat gezeigt, daß Von Rad in seiner Theologie verschiedene Denkmodelle miteinander kombiniert: das verheißungsgeschichtliche Modell, das überlieferungsgeschichtliche Modell und das heilsgeschichtliche Modell.

Goppelt greift diese Linie auf und führt sie weiter. Der Begriff 'Wirkungsgeschichte' steht zentral in den Theologien von beiden Von Rad und Goppelt. In der Theologie Von Rads gehört die Kenntnis der Wirkungsgeschichte zum vollen Verständnis eines Textes. Die Funktion und Wichtigkeit dieses Begriffs in der Theologie Goppelts werde ich gleich ausführlicher behandeln.

Goppelt versucht in seiner Theologie, zuerst mit einem historisch fesistellbaren Minimum zu arbeiten. Von Rad (1966: 120) unterscheidet seiner seit zwischen einem kritisch gesicherten Minimum und einem theologischen Maximum. Er ist der Meinung, da $B$ eine Theologie nicht nur mit einem kritisch gesicherten Minimum arbeiten kann.

Goppelt führt nicht eine Heilsgeschichte vom Alten Testament her weiter. Er füllt die Heilsgeschichte mit einem neuen Inhalt durch Reflexion auf dem irdischen Jesus.

2.3 Der Ansatz zur neutestamentlichen Theologie

Goppelt (1985: 54) stellt die Frage: 'Was ist der Ansatz zur Ntl. Theologie und 
damit die Basis der Weltreligion des Christentums?' Er lenkt die Aufmerksamkeit darauf, da $B$ eine Untersuchung der Struktur der Evangelien zeigt, daß sie gleichsam vom Ende, von Passion und Ostern her geschrieben sind. Zur Bildung christlicher Gemeinden und damit zu einem Weiterwirken Jesu kam es nach den früchchristlichen Überlieferungen erst durch das Osterkerygma: '... dieses ist der Ansatz der Neutestamentlichen Theologie' (1985: 56). Die neutestamentliche Theologie fragt nach Jesus, wie er sich den Nachfolgenden in den Erdentagen darbot, und das ist auch der Jesus, der geschichtlich weiterwirkte (Goppelt 1985: 58; dagegen Perrin 1984: 415-419).

\subsubsection{Die traditionskritische Analyse bei Goppelt}

Goppelt ist der Meinung, $\mathrm{da} B$ er seine Theologie des Neuen Testaments auf eine eigene traditionskritische Analyse stellen muß (1985: 64). Er verfährt dabei in folgender Weise: 1 . Er versucht erst negativ abzuheben, was sich als sekundär erweist. 2. Er versucht dann von einem Minimum an Echtheit aus durch verstehende Deutung ein Gesamtbild aufzubauen. Das Minimum an Echtheit ergibt sich nach dem Kriterium der religionsgeschichtlichen Besonderheit sowohl gegenüber der Umwelt als auch gegenüber der frühchristlichen Gemeinde. Die Anwendung dieses Kriteriums wird unterstützt, wenn Überlieferungen von mehreren untereinander unabhängigen Quellen, z B Markus und $Q$ von der synoptischen Tradition und von Paulus, geboten werden. Goppelt lenkt auch die Aufmerksamkeit auf die sprachlichen und stilistischen Merkmale, die dem Kern der Jesusüberlieferung eigen sind, die J Jeremias (1971: 14-49) herausgearbeitet hat.

Wir können sagen, da $B$ wir bei Goppelt etwas wie eine 'Theologie Jesu' finden. $\mathrm{Da} B$ der Schwerpunkt für Goppelt hier liegt, kann man darin sehen, daß der erste Hauptteil seiner Theologie, 'Jesu Wirken in seiner theologischen Bedeutung', Inhalt des ganzen ersten Bandes ist. (Den zweiten, dritten und vierten Hauptteil findet man in Band 2.)

2.3.2 Das Verhāltnis zwischen dem irdischen Jesus und dem auferstandenen Christus, dem zu verkündigenden und dem verkündigten Jesus

Aus dem, was bisher gesagt wurde, wird deutlich, daß wir unser Augenmerk auf dieses Kernproblem der neutestamentlichen Theologie legen müssen.

Ferdinand Hahn (1982: 101) weist darauf hin, daß die eschatologische Wirklichkeit des Heils sich in irdisch-geschichtlicher Gestalt ereignet hat, wodurch Kontinuität gestiftet und begründet wurde, die über Kreuz, Auferweckung und Geistausgießung hinaus auf die zukünftige Vollendung verweist. Jedoch handelt es sich um eine Kontinuität, die innergeschichtlich nicht vorhanden und möglich ist, die 
vielmehr mit Jesu Auferweckung von Gott gewährt wurde und weiterhin von ihm gewährt und gewahrt wird.

Wenn dies aber gilt, meint Hahn, dann kann eine neutestamentliche Theologie auf Jesu Handeln und Verkündigen weder verzichten noch eine diesbezügliche Darstellung verselbständigen. In den Lehrbüchern Rudolf Bultmanns und Joachim Jeremias liegen in sehr charakteristischer Weise alternative Positionen vor. Hatte Bultmann auf die Integration der Verkündigung Jesu verzichtet und sie den Voraussetzungen einer Theologie des Neuen Testaments zugeordnet, so hat umgekehrt Jeremias die Verkündigung Jesu mit aller Eindeutigkeit als den eigentlich maßgebenden Bestand urchristlicher Überlieferung von jeder nachösterlichen Gemeindetradition abgehoben. Aber auch eine vermittelnde und in mancher Hinsicht weiterführende Position wie die von Leonhard Goppelt, meint Hahn (1982: 102), bleibt bei einer Verselbständigung des Wirkens Jesu stehen.

Ergebnisse, die auf dem Wege historisch - kritischer Analyse gewonnen werden, verweisen uns auf die geschichtliche Einmaligkeit der göttlichen Heilsvenwirklichung. Sie konkretisiert die Heilszusage im Blick auf die historische Situation, in der Jesus gelebt, geredet und gehandelt hat. Das ist theologisch deshalb von Belang, weil die Realität der Inkarnation nicht in irgendeiner Weise doketisierend überspielt werden darf (Hahn 1982: 104).

Aber noch wichtiger und bedeutsamer ist der durch die historische Analyse sichtbar werdende Rezeptionsvorgang selbst. Es zeigt sich sehr deutlich, wie frühe theologische Reflexion die neugewonnene Einheit von vorgegebener Jesustradition und grundlegendem Bekenntnis miteinander in Beziehung setzte. Die Rückfrage nach Jesus hat theologisch nur Bedeutung, só Hahn, wenn sie mit der Rezeptionsfrage verbunden bleibt.

Die Rezeptionsfrage kommt meines Erachtens implizit wohl im zweiten Band der Theologie Goppelts zur Sprache. In seinem Vorwort zum 2. Band schreibt Jürgen Roloff, daß Goppelt uns dazu ermutigt, hinter der verwirrenden Vielfalt der theologischen Positionen das apostolische Christuszeugnis zu suchen, um es als alleinige Norm christlichen Glaubens und Lebens verstehend auf die gegenwärtige Wirklichkeit hin zu entfalten.

2.3.3 Goppelt und die historische Problematik des Ansatzes einer neutestamentlichen Theologie

Es sei daran erinnert, daß die Frage nach dem Verhältnis zwischen dem verkündigenden und dem verkündigten Jesus eine moderne Frage ist. Für die Aufklärungstheologie ist nur das theologisch wichtig, was auch historisch anweisbar ist. 
Goppelt (1985: 58-59) setzt sich deutlich mit dieser Problematik auseinander und weist darauf hin, daB Ferdinand Christian Baur die historische Problematik des Ansatzes der neutestamentlichen Theologie bewußt machte, indem er den Grundsatz herausstellte, der fortan die rein historische Schriftforschung bestimmte: Baur behauptete, da $\mathrm{B}$ es historischem Denken widerspreche, eine geschichtliche Entwicklung, das Werden des Christentums, von einem Mirakel, nämlich von der Auferstehung, abzuleiten und darin die Basis des Christentums zu sehen. Historisch faßbar sei nur zweierlei, nämlich das historische Hervortreten Jesu und anderseits der Osterglaube der Jünger. Deshalb, sagt Goppelt (1985: 59), suchte die rein historische Forschung bald in ersterem, bald in letzterem den Ansatz. Im Rationalismus und in der liberalen Theologie, der auch die religionsgeschichtliche Schule verpflichtet blieb, fand man den entscheidenden Ansatz beim historischen Jesus. Hier können die Namen Johannes Weiß, Wilhelm Bousset und Herbert Braun genannt werden.

Diese Reduktion des Ansatzes auf den historischen Jesus wurde aber in der folgenden Generation mit Nachdruck als historische Fiktion abgelehnt. Der historische Ausgangspunkt für die gesamte Entwicklung sei eindeutig der Osterglaube. Hier können die Namen von Ferdinand Christian Baur und Rudolf Bultmann genannt werden.

Goppelt (1985: 61-62) lenkt aber die Aufmerksamkeit darauf, daß das Osterkerygma in der jüdischen Umwelt Jesu schlechthin einzigartig war. Keine der vergleichbaren antiken Gestalten wirkt durch die Botschaft von ihrer Auferstehung weiter. Die entscheidende Frage ist für Goppelt, ob das Erdenwirken Jesu tatsächlich der sachliche - und nicht etwa nur ein psychologischer - Grund des Osterkerygmas ist. Führt Jesu Wirken seiner sachlichen Struktur nach zur Passion und Auferstehung? Wegen dieser Überlegungen ist Goppelt der Meinung, daß es nicht nur um der eige-nen Denkstruktur des Neuen Testaments willen, sondern auch aus historischen Gründen angemessen ist, die Darstellung der neutestamentlichen Theologie mit der des Wirkens und Weges Jesu zu beginnen.

Wenn man diesen Ansatz von Goppelt ernst nimmt, verliert die Kritik gegen ihn meines Erachtens viel von ihrem Gewicht.

\section{ZUSAMMENFASSUNG}

In diesem Artikel wurde versucht nachzuweisen, daß Cullmann die Heilsgeschichte zur tragenden, aber nicht unbestrittenen Grundlage einer neutestamentlichen Theologie erhob (auch Merk 1980: 464). Obwohl mit Recht gesagt werden kann, daß Cullmann sich nicht am ganzen Neuen Testament orientiere, sondern nur an 
einem besonderen Aspekt, eröffnete und betonte er meines Erachtens jedoch eine wichtige Perspektive, die als ein Ansatzpunkt für eine Theologie des Neuen Testaments nicht aus den Augen verloren werden darf.

Goppelt suchte seinen hermeneutischen Ansatz in Weiterführung der heilsgeschichtlichen Fragestellung. Sehr nuanciert füllte er die Heilsgeschichte durch Reflexion auf den irdischen Jesus mit einem neuen Inhalt. Der Einsatz seiner neutestamentlichen Theologie ist nicht ein allgemeines Osterkerygma, sondern die theologische und damit zu interpretierende Entfaltung von Jesu Tat und Wort (Berger 1988: 361; Merk 1980: 466). Obwohl die Theologie Goppelts wegen seines frühen Todes unvollendet geblieben ist, "kann doch der 1. Band der Darstellung Goppelts als die "zur Zeit ... beste und zuverlässigste wissenschaftliche Darstellung der Verkündigung und Wirksamkeit Jesu" angesehen werden' (Merk 1980: 466).

\section{Literaturverzeichnis}

BERGER, K 1988. Neutestamentliche Theologien. $T h R$ 53, 354-370.

BULTMANN, R 1967. Heilsgeschichte und Geschichte: Zu Oscar Cullmann, Christus und die Zeit, in Dinkler, E (Hrsg), Exegetica: Aufsätze zur Erforschung des Neuen Testaments, 356-368. Tübingen: Mohr.

CLEMONS, JT 1972. Critics and criticisms of salvation history. Rellife 41, 89-100.

CONZELMANN, H 1964. Die Mitte der Zeit: Studien zur Theologie des Lukas. 5. Aufl. Tübingen: Mohr. (BHTh 17.)

CULLMANN, O 1967. Heil als Geschichte: Heilsgeschichtliche Existenz im Neuen Testament. 2. Aufl. Tübingen: Mohr.

CULLMANN, O 1975. Die Christologie des Neuen Testaments. 5. Aufl. Tübingen: Mohr.

FUCHS, E 1960. Christus das Ende der Geschichte, in Fuchs, E, Zur Frage nach dem historischen Jesus: Gesammelte Aufsätze II, 79-99. Tübingen: Mohr.

GOPPELT, L 1985. Theologie des Neuen Testaments, hrsg von Jürgen Roloff. 3. Aufl. Nachdruck. Göttingen: Vandenhoeck. (Uni-Taschenbücher 850.)

HAHN, F 1982. Urchristliche Lehre und neutestamentliche Theologie: Exegetische und fundamentaltheologische Überlegungen zum Problem christlicher Lehre, in Kern, W (Hrsg), Die Theologie und das Lehramt, 63-115. Freiburg: Herder. (Questiones Disputatae 91.)

HASEL, G 1978. New Testament Theology: Basic issues in the current debate. Grand Rapids: Eerdmans.

HESSE, F 1971. Abschied von der Heilsgeschichte. Zürich: Theologischer Verlag. (ThSt 108.) 
JEREMIAS, J 1971. Neutestamentliche Theologie, Erster Teil: Die Verkündigung Jesu. Gütersloh: Gütersloher Verlagshaus Gerd Mohn.

JüNGEL, E 1985. Glauben und Verstehen: Zum Theologiebegriff Rudolf Bultmanns. Heidelberg: Carl Winter, Universitätsverlag. (SHAW 1.)

KäSEMANN, E 1972. Paulinische Perspektiven. 2. Aufl. Tübingen: Mohr.

KASPER, W 1970. Glaube und Geschichte. Mainz: Matthias-Grünewald-Verlag.

KLEIN, G 1971. Bibel und Heilsgeschichte: Die Fragwürdigkeit einer Idee. ZNW 62,1-47.

KüMMEL, WG 1978. Heilsgeschichte im Neuen Testament? in Grässer, E \& Merk, O (Hrsg), Heilsgeschehen und Geschichte, Band 2: Gesammelte Aufsätze 1965-1977. Marburg: Elwert. (MThSt 16.)

LADD, GE 1971. The search for perspective. Interp. 25,41-62.

MERK, O 1972. Biblische Theologie des Neuen Testaments in ihrer Anfangszeit: Ihre methodischen Probleme bei Johann Philipp Gabler und Georg Lorenz Bauer und deren Nachwirkungen. Marburg: Elwert. (MThSt 9.)

MERK, O 1980. s v Biblische Theologie II. TRE.

OEMING, M 1985. Gesamtbiblische Theologien der Gegenwart: Das Verhältnis von AT und NT in der hermeneutischen Diskussion seit Gerhard von Rad. Stuttgart: Kohlhammer.

PERRIN, N 1984. Jesus and the Theology of the New Testament. JR 64, 413-431.

SCHNACKENBURG, R 1963. Neutestamentliche Theologie: Der Stand der Forschung. München: Kösel. (BiH 1.)

VIELHAUER, P 1965. Zum 'Paulinismus' der Apostelgeschichte, in Vielhauer, P, Aufsätze zum Neuen Testament, 9-27. München: Chr Kaiser Verlag. (TB 31.)

VON RAD, G 1966-1968. Theologie des Alten Testaments. 2 Bände. 5. Aufl. München: Chr Kaiser. 